\title{
EXPERIMENTAL EVALUATION OF ENERGY PARAMETERS OF VOLUMETRIC VIBROSEPARATION OF BULK FEED FROM GRAIN
}

\author{
Igor Palamarchuk ${ }^{1}$, Ivan Rogogvskiii ${ }^{1}$, Liudmyla Titova ${ }^{1}$, Oleg Omelyanov ${ }^{2}$ \\ ${ }^{1}$ National University of Life and Environmental Sciences of Ukraine, Ukraine; \\ ${ }^{2}$ Vinnytsia National Agrarian University, Ukraine \\ vibroprocessing@gmail.com,rogovskii@nubip.edu.ua,ludmylkatitova@gmail.com,oon@vsau.vin.ua
}

\begin{abstract}
Among the main directions of intensification of vibrating technological action in the separation of bulk agricultural products can be noted not only the adjustment of amplitude-frequency characteristics, but also the improvement of dimensions and structural parameters of drive mechanisms. The developed scheme of the studied process of vibro screening provides an inclined location of the working tank, which allows to realize the formation of spatial oscillations of the sieve surface. The developed scheme of vibrating screen process provides an inclined location (arrangement) of the working tank, which allows to accomplish the formation of spatial oscillation of the sieve surface. An experimental model of the screen of spatial oscillations was developed and the conducted research allowed to obtain graphical dependences of energy consumption on the processing of crushed grain material on the change of mass, geometric and structural parameters of the oscillatory system. For the choice of the effective mode of vibratory screening of combined feeds, the evaluation criteria were the eccentricity of the drive shaft, the angle of the sieve surface, the amount of energy consumption and patterns of its change in terms of variation of the force pulse when changing the eccentricity of the drive shaft, the measurability of oscillating motion from flat to spatial with appropriate adjustment of the design parameters. Experimental studies have revealed the effectiveness of the use of spatial oscillations of the sieve surface in the separation of bulk masses, allowed to substantiate the design parameters of the vibrating screen.
\end{abstract}

Keywords: raw material, seed, vibrating screen, vibration drive.

\section{Introduction}

The most complete theoretical studies of the operation of sieve surfaces that implement longitudinal oscillating motions are performed in the fundamental works $[1 ; 2]$. The first recorded a system of differential equations of spatial motion of the operating device of a vibrating machine with several mechanical vibrators, the axes of which are arbitrarily oriented in space [3; 4]. The problem of moving agricultural materials on the working surfaces of the separators was solved, provided that such materials are discrete solids; tasks were solved of layer-by-layer processes and self-sorting, clogging and cleaning of sieve openings, sifting of seeds through sieve openings. In the works $[5 ; 6]$ the dependences of the quality of separation of a flat sieve on the main parameters are obtained: kinematic mode of operation and angle of inclination of the sieve to the horizon, angle of oscillation, shape and location of holes, sieve sizes and specific loading, humidity and clogging of grain material [7].

Kalivoda J. [8] determined the value of the critical velocity of sifting particles through the holes of the sieve, at an angle of inclination of the latter not more than $10^{\circ}$ in the direction of particle rise, taking into account air resistance.

Golovin A. [9] found that biharmonic oscillations of the sieve are one of the effective ways to improve the technological process of grain separation. He described the movement of grain material on the sieve, which performs biharmonic oscillations, developed a method for calculating the parameters of the eccentric drive mechanism of the sieve with an elliptical pulley, which obtained the biharmonic law of its oscillations, proposed and substantiated the separator scheme with biharmonic flat sieve drive.

Bahadirov G. [10] developed methods for calculating the intensification of vibrocentric separation processes according to technological indicators of productivity and quality, created and introduced into serial production dynamic, surface and volume intensifiers that increase productivity and quality of grain material separation by vibrocentric separators.

Astanakulov K. [11] found that the quality of the process of separation of grain raw materials on the non-perforated friction oscillating surface significantly depends on the structural and kinematic parameters of the vibrating grain cleaning machine: amplitude, frequency and angle of oscillation, as well as angles of inclination of the operating device to the horizon in transverse directions, as a result he substantiated the rational design and kinematic parameters and the actual working devices of the vibrating grain cleaning machine for processing wheat, barley, oats and rye. 
Bulgakov V. proposed to intensify the process of separation of grain material at the sieve state due to the different angle of installation of the upper and lower sieves relative to the direction of the exciting force [12].

Elfverson C. [13] and Petre I. Miu [14] claim that the vibrating sieve separators of the traditional scheme of separation of grain mixtures by sieves have practically reached the limit of improvement and further increase of productivity is realized by increasing the working area of sieves. They investigated the influence of kinematic, technological and structural parameters on interlayer processes, the speed of particle movement in the grain layer and along the sieve surface in the presence of passive leavening agents.

The aim of the research is to experimentally evaluate the influence of geometric parameters of the driving mechanism of the vibrating screen of bulk combined fodder from masses of crushed grain raw materials under the conditions of spatial oscillating motion of sieve surfaces on energy consumption to create and maintain the processing.

\section{Materials and methods}

To achieve this aim, the following tasks were provided: development of basic schemes of vibrating screen and drive mechanism for providing its working devices with spatial oscillations; according to the presented schemes development of experimental installation of a vibrating screen for separation of bulk combined fodder from the crushed grain components; obtaining graphical dependences that reflect the influence of design parameters of the developed vibrating screen on the change of energy consumption on the implementation of the studied separation process.

During the experimental analysis and substantiation of energy regime characteristics of the developed vibrating screen (Fig. 1) with a sieve surface that performs spatial oscillations German Robotron equipment and special electrical devices were used to determine energy and amplitudefrequency characteristics, methods of mathematical analysis and their processing in mathematical MathCAD environment to obtain the necessary graphical dependencies. When adjusting the parameters of the volumetric vibrations of the sieve and the intensity of the oscillating action in a given plane, the angle of inclination of the operating devices of the screen was changed, which was carried out with the help of a specially designed sleeve.

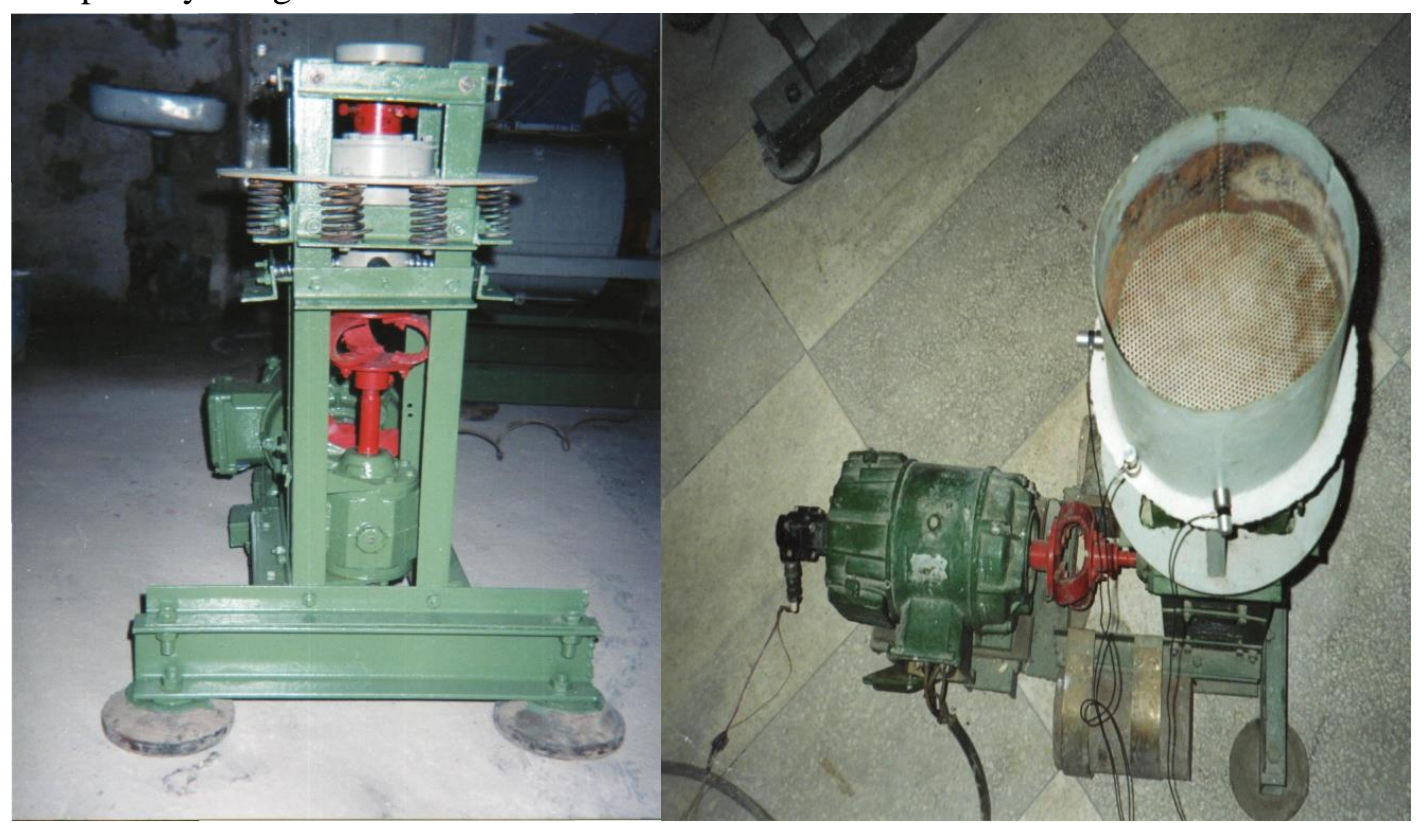

Fig. 1. Photos of developed experimental installation of vibrating screen and the drive mechanism of volume oscillations

The structure of the bulk material of feed consisted of crushed mass of wheat (45\%), corn (30\%), sunflower meal (8\%), soybean meal (12\%), premix (5\%), which were completed on 5,10, 15 and $20 \mathrm{~kg}$. 
In the process of vibroseparation it was acceptable: the size of loose feed up to $3 \mathrm{~mm}$, and the residue on the sieve did not exceed $10 \%$.

The developed vibrating screen consists of a frame 9 (Fig. 2), on the base plate of which a vibrating platform 6 is mounted in a circle through cylindrical springs 7 , on which a sieve is mounted, which can have one or several conical or cylindrical sieves 4 . To remove the separated fractions from the machine there is a sloping cone 5 (one or more depending on the number of sieves) and launders 8 . In the lower part of the apparatus a single-shaft vibrator 10 is attached to the vibrating table, which is driven by a DC motor. In the upper part of the machine the hopper 1 is mounted on supports 2, which has an adjusting damper 3 for regulating the supply of products to the working space of the machine.

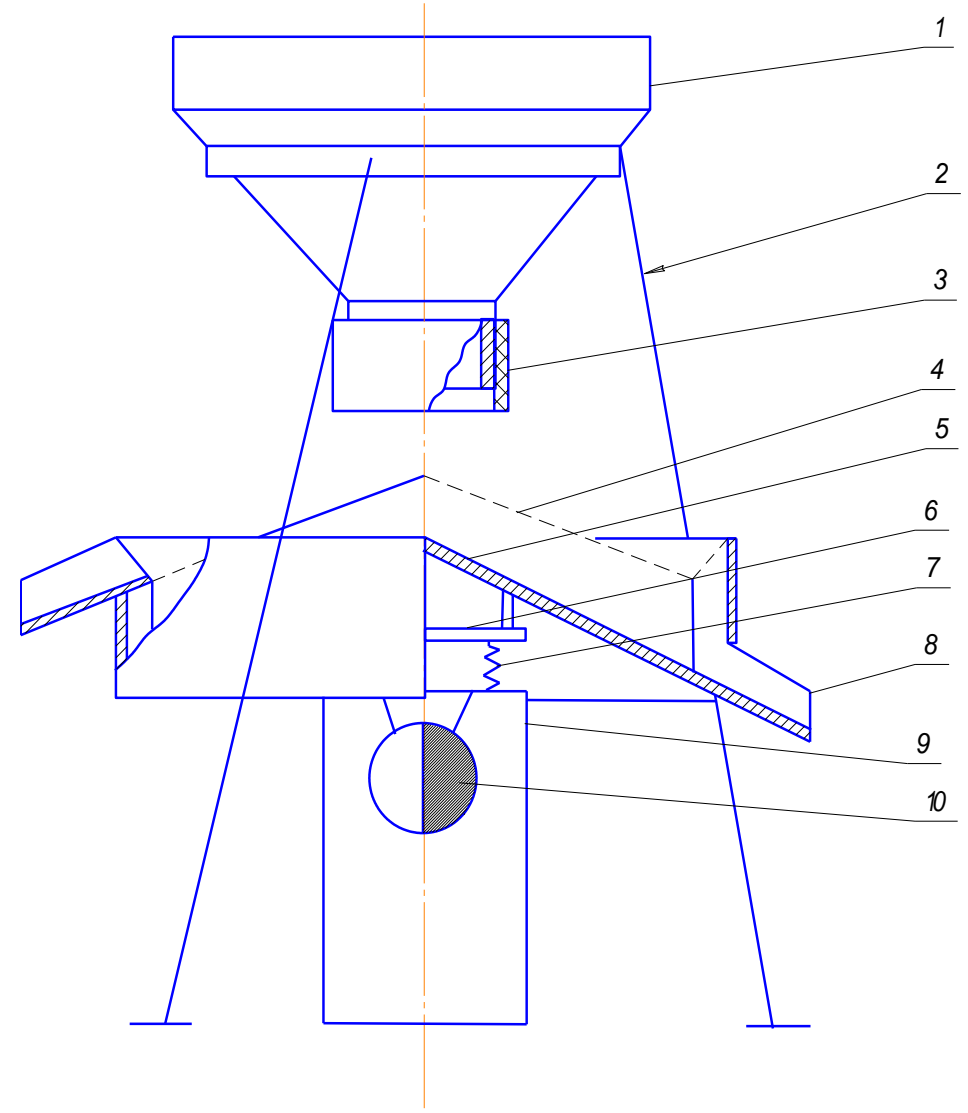

Fig. 2. Scheme of vibrating screen with conical sieve surface: 1 - bunker; 2 - support; 3 -damper; 4 - sieve; 5 - sloping cone; 6 - vibroplatform; 7 - elastic element; 8 -launder; 9 -machine bed; 10 - vibrating exciter

The vibrating exciter of this screen (Fig. 3) contains a drive of the vertical shaft 3, consisting of an electric motor 5 , a conical reductor 6 and a sleeve 9, which is mounted on the shaft and kinematically connected to the separator through bearings 8 . Placement of the sleeve on the shaft with eccentricity, and also execution of its outer surface in the form of a cylinder, the axis of which is with the axis of the shaft 3 - an acute angle $\varphi$, provide spatial oscillations of the working bodies of the machine.

The drive of the shaft 3 (Fig. 3) from the motor 5 is carried out through the conical reductor 6 . The inner rings of the bearings 8 are pressed on the sleeve 9 , which is located on the shaft, so they rotate with it. The outer rings of the bearings 8 , and with them the vibrating platform 1 and the separator itself do not rotate, but only move in the radial and vertical directions. The peculiarity of this vibratory drive is that in the horizontal plane the outer bearing races 8 and the separator perform a gyration movement, i.e. a gradual circular motion with a radius equal to the eccentricity of the inner surface of the sleeve relative to the axis of rotation of the shaft 3 . The springs 2 , which are fixed at one end in a stated support, limit the rotation of the separator relative to its own axis. In addition, since the axis of the outer surface of the sleeve is inclined relative to the axis of the shaft at an angle $\varphi$, the outer race of the bearing and the separator perform oscillating motion in the vertical plane, which allows to adjust the oscillation amplitude of the machine in a wide range. In addition, the installation of the shaft 3 on the frame by 
means of bearings with adjustable elastic stops 6 allows to reduce the dynamic loads on all bearings of the drive and increase their service life.

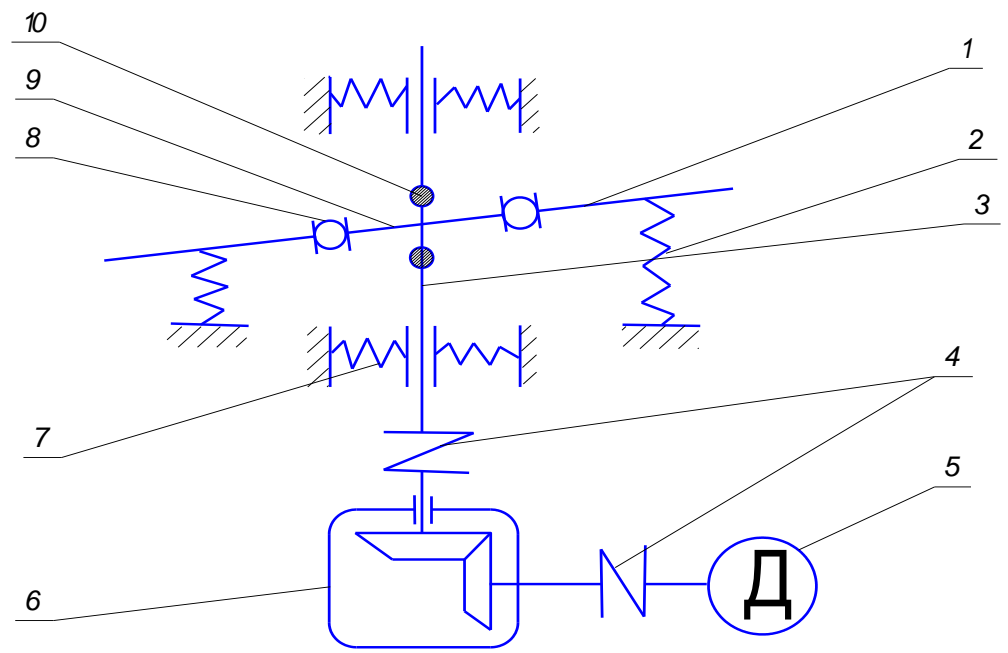

Fig. 3. Scheme of the vibrating drive of the screen: 1 - vibroplatform; 2,7 - elastic elements;

3 - shaft; 4 - coupling; 5 - electric motor; 6 - conical reductor; 8 - bearing assembly;

9 - sleeve; 10 - counterweight

\section{Results and discussion}

According to the results of experimental studies, the following energy dependences were obtained (Fig. 4 - Fig. 7). When increasing the load weight of the working container from 5 to $20 \mathrm{~kg}$, the drive power of the machine changes by $14 \%$ (Fig. 4), with more stable operation of the drive motor observed at higher loads of raw materials.

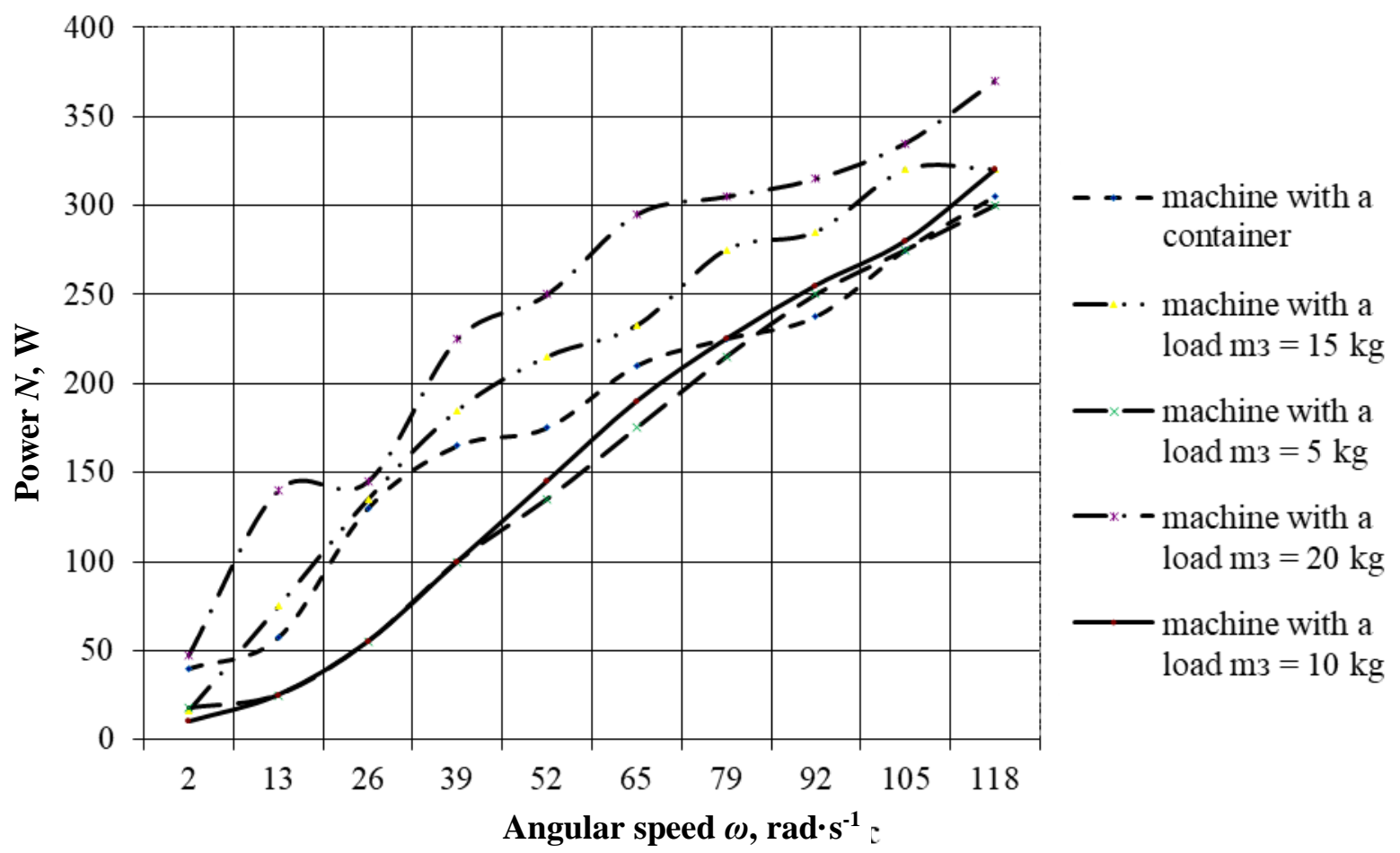

Fig. 4. Graph of dependence of power consumption $N$ on the angular speed of the drive shaft of the screen $w$ when changing the load mass of the working tank

Figure 5 shows the graphical dependences of the power $N_{i}$ on the angular velocity $\omega$ with a compatible change in the angle of the axis of the drive shaft $\varphi$ and the value of its eccentricity e. Changing the angle of the drive shaft axis $\varphi$ from 0 leads to spatial oscillations in the studied system, which improves the separation conditions and with increasing energy consumption for the process 
decreases. As the value of the eccentricity $e$ of the drive shaft of the vibrator increases, the amplitude of oscillations increases and, accordingly, the permeability of vibrations in the mass of the bulk medium increases.

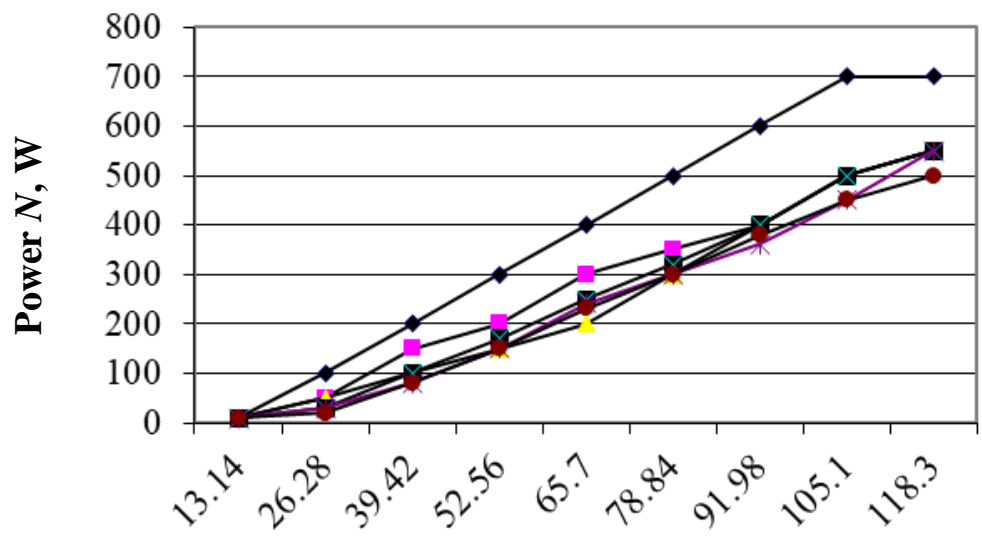

$$
\begin{aligned}
& \multimap \varphi \circ=0, \text { eo }=0 \\
& -\varphi 1=1, \mathrm{e} 1=0.75 \mathrm{~mm} \\
& -\varphi 2=4 \text {, e2 }=1.5 \mathrm{~mm} \\
& \rightarrow-\varphi 3=7, \text { e } 3=2.25 \mathrm{~mm} \\
& \text { 米 } \varphi 4=10, \mathrm{e} 4=3 \mathrm{~mm} \\
& \multimap \varphi 5=13, \text { e } 5=3.75 \mathrm{~mm}
\end{aligned}
$$

Angular speed $\omega, \operatorname{rad} \cdot \mathrm{s}^{-1}$

Fig. 5. Graph of dependence of the power $N$ and the angular speed $\omega$ when changing the angle of the axis of the drive shaft $\varphi$ and the value of its eccentricity $e$

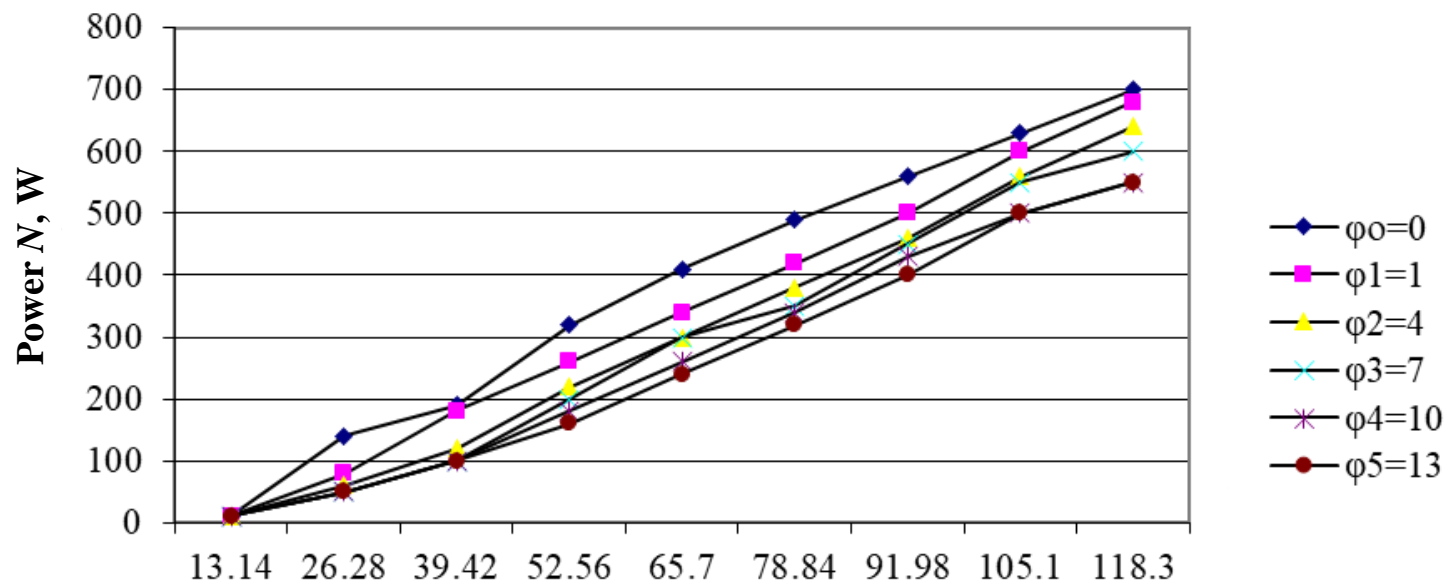

Angular speed $\omega, \operatorname{rad} \cdot \mathrm{s}^{-1}$

Fig. 6. Graph of the power $N$ and the angular velocity of the drive shaft of the screen $\omega$ when changing the angle of the shaft axis $\varphi$
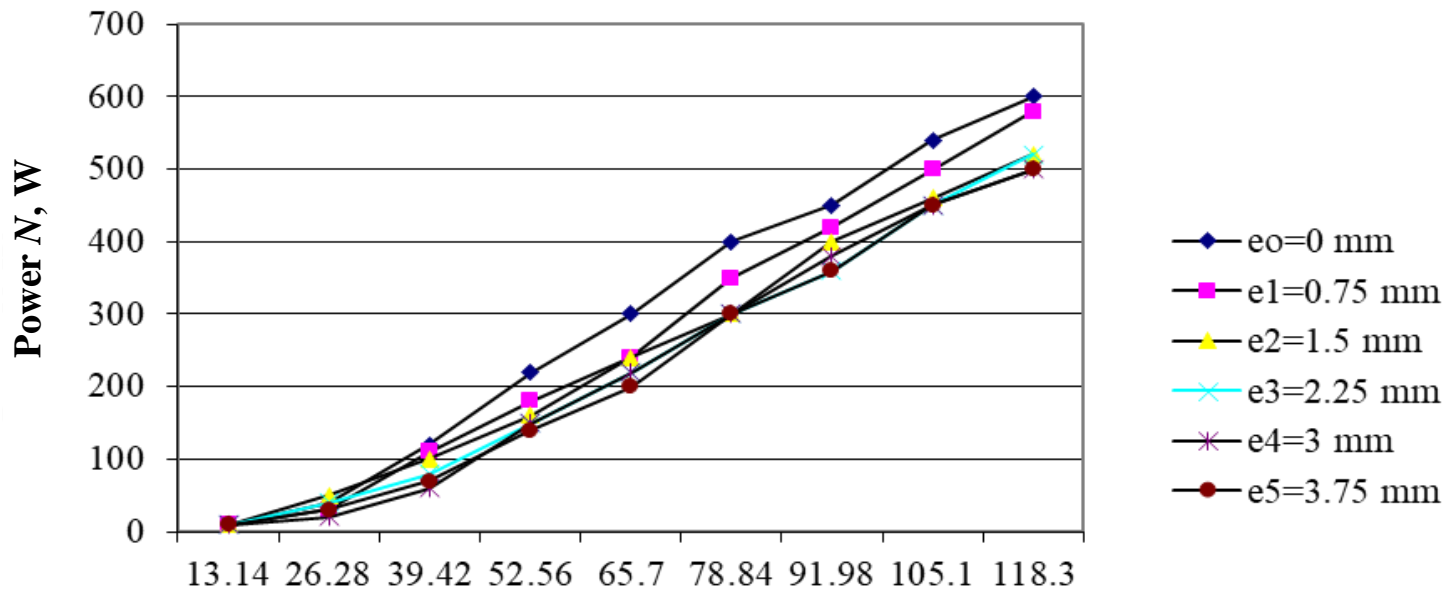

Angular speed $\omega, \operatorname{rad} \cdot \mathrm{s}^{-1}$

Fig. 7. Graph of dependence of the power $N$ and the angular velocity $\omega$ when changing the value of the eccentricity of the drive shaft of the screen $e$ 
Figure 6 shows the graphical dependences of the power $N_{i}$ on the angular velocity $\omega$ only when changing the angle of the drive shaft axis $\varphi$, which confirms the effect of reducing energy consumption to create spatial motion of the working bodies of the machine. Figure 7 shows the graphical dependences of the power $N_{i}$ on the angular velocity $\omega$ only when the value of its eccentricity $e$ changes from 0 , which leads to the creation of kinematic vibration excitation, which reduces the oscillating masses in the system and reduces the energy consumption for the process.

At the value of the angle of the axis of the drive shaft of the screen $\varphi=0^{\circ}$ and the value of its eccentricity $e=0 \mathrm{~mm}$, there is a transfer of the container of flat oscillations, which increases the energy consumption by $28.6 \%$ compared to spatial oscillations (Fig. 5). The rest presented in Fig. 5 operating parameters of the vibrating screen correspond to the transmission of spatial oscillations, in which energy consumption is reduced by $24 \%$. When changing the angle of the drive shaft axis $\varphi$ by 130 , the energy consumption for the separation process changes by $21.4 \%$ (Fig. 6), and when changing the value of the eccentricity of the drive shaft of the screen $e$ by $3.75 \mathrm{~mm}$, the energy consumption decreases by $16.7 \%$ (Fig. 7).

One of the progressive directions in the use of low-frequency mechanical oscillations for separation of bulk agricultural products is creation of devices with operating devices of spatial oscillatory action, the intensity of which depends not only on the amplitude and frequency of their source, but also on the method of excitation, transmission of oscillations and installation geometry. The developed experimental setup of the vibrating screen allowed to model the regimes of separation of bulk feed during the transmission of flat and spatial oscillations, changing the eccentricity of the drive shaft and the amplitude of oscillations, varying the angle of the working screen and the geometry of oscillating motions.

\section{Conclusions}

1. Energy consumption during separation in the conditions of flat oscillations increases by $28.6 \%$ in comparison with the modes of realization of the spatial motion of sieve surfaces.

2. Energy consumption for the separation process changes more significantly when changing the geometry of oscillatory motions by $21.4 \%$ than when changing the amplitude of oscillations by $16.7 \%$. With increasing load weight of the working container, the operation of the drive mechanism is more stable, although there is an increase in the energy consumption by $14 \%$.

\section{References}

[1] Triana C., Fajardo F. Experimental study of simple harmonic motion of a spring-mass system as a function of spring diameter. Revista Brasileira de Ensino de Física. 2013. Vol. 35. No 4. pp. 18-36. DOI: $10.1590 / \mathrm{S} 1806-11172013000400005$.

[2] Adams D.E. Mechanical vibrations. Perdu University. USA. 2010. 131 p. [online] [21.03.2021] Available at: https://engineering.purdue.edu/ deadams/ME563/notes_10.pdf.

[3] Czubak P. Vibratory conveyor of the controlled transport velocity with the possibility of the reversal operations. Journal of Vibroengineering. 2016. Vol. 18. Issue 6. pp. 3539-3547. DOI: $10.21595 /$ jve.2016.17257.

[4] Nazarenko I., Dedov O., Bernyk I., Rogovskii I., Bondarenko A., Zapryvoda A., Titova L. Study of stability of modes and parameters of motion of vibrating machines for technological purpose. Eastern-European Journal of Enterprise Technologies. 2020. Vol. 6 (7-108). pp. 71-79. DOI: $10.15587 / 1729-4061.2020 .217747$.

[5] Wang D., Zhao C., Yao H. Vibration synchronization of a vibrating system driven by two motors. Advances in Vibration Engineering. 2012. Vol. 11. Issue 1. pp. 59-73.

[6] Michalczyk J. Phenomenon of force impulse restitution in collision modelling. Journal of Theoretical and Applied Mechanics. 2008. Vol. 46. Issue 4. pp. 897-908.

[7] Rogovskii I.L., Delembovskyi M.M., Voinash S.A., Scherbakov A.P., Teterina I.A., Sokolova V.A. Reliability indexes of vibrating platforms for compaction of construction mixtures. IOP Conference Series: Materials Science and Engineering. 2021. Vol. 1047. pp. 012026. DOI: 10.1088/1757899X/1047/1/012026.

[8] Kalivoda Julie. Effect of sieving methodolgy on determining particle size of ground corn, sorghum, and wheat. Southeast Community College-Beatrice, Kansas State University. A thesis submitted in 
partial fulfillment of the requirements for the degree Master of Science Department of Grain Science College of Agriculture. Kansas State University Manhattan, Kansas. 2016. 107 p. [online] [21.03.2021] Available at: https://core.ac.uk/download/pdf/33381154.pdf.

[9] Golovin A.Yu., Chupin P.V., Soyunov A.S., Prokopov S.P., Abdylmanova R.H. Comparative analysis of operation indicators of flat sieve vibrating in horizontal plane. IOP Conf. Series: Earth and Environmental Science. 2021. Vol. 659. pp. 012047. DOI: 10.1088/1755-1315/659/1/012047.

[10] Bahadirov G., Barakaev N. Study of grain motion parameters on a sloping shelf. Applied Mechanics and Materials. 2015. Vol. 809-810. pp. 51-56.

[11] Astanakulov K.D., Karimov Yo.Z., Fozilov G.P. Design of a grain cleaning machine for small farms ama, Agricultural Mechanization in Asia, Africa \& Latin America. 2011. Vol. 42. pp. 37-40.

[12] Bulgakov V., Sevostianov I., Kaletnik G., Holovach I., Ihnatiev Y. Theoretical studies of the vibration process of the dryer for waste of food. Rural Sustainability Research. 2020. Vol. 44(339). pp. 32-45.

[13] Elfverson C., Regner S. Comparative precision of grain sieve and pneumatic classification on a kernel level. Applied Engineering in Agriculture. 2020. Vol. 16 (5). pp. 537-541.

[14] Petre I. Miu, Heinz-Dieter Kutzbach. Modeling and simulation of grain threshing and separation in threshing units. Computers and Electronics in Agriculture. 2018. Vol. 60. pp. 96-104. 